\title{
Modified Harrington Procedure for Acetabular Insuficiency Due to Metastatic Malignant Disease
}

\author{
WI Faisham, MMed Ortho, DAJ Muslim, MRCS, VMK Bhavaraju*, MD, AH Nawaz, MMed Ortho, \\ W Zulmi, MS Ortho \\ Department of Orthopaedic, School of Medical Science of Universiti Sains Malaysia, Kota Bahru, Malaysia \\ *Department of Nuclear Medicine, Radiotherapy \& Oncology, School of Medical Science of Universiti Sains \\ Malaysia, Kota Bahru, Malaysia
}

\begin{abstract}
Extensive peri-acetabular osteolysis caused by malignant disease process is a major surgical challenge as conventional hip arthroplasty is not adequate. We describe a modified use of the Harrington procedure for acetabular insufficiency secondary to metastatic disease in twelve patients. The procedures include application of multiple threaded pins to bridge the acetabular columns, anti-protrusio cage and cemented acetabular cup. Eleven patients were able to walk pain free and achieved a mean Musculoskeletal Tumour Society Functional Score of 80 (range, 68 to 86).
\end{abstract}

Key Words:

Acetabular metastases, Harrington procedure

\section{INTRODUCTION}

Metastatic bone disease is common in cancer patients and causes substantial disease-related morbidity and mortality ${ }^{1,2}$. Treatment for bone metastases is usually palliative, with goals of orthopaedic management being adequate control of pain, prevention or stabilization of pathological fractures, improvement of function and ambulation, facilitation of medical and nursing care, and improvement of psychological well-being.

The proximal femur and periacetabular region are the most common sites of involvement in the appendicular skeleton $1,2,3$. This is also a common site of pathological fracture because of the significant forces that pass through this region while standing. A fracture involving the acetabular region may cause severe pain and result in impaired mobility and function. Palliative radiotherapy is a well-established treatment to control the bone pain from metastasis. However, when it comes to weight bearing and ambulation, surgical intervention is required to stabilize the joint ${ }^{4}$.

\section{MATERIALS AND METHODS}

Between January 2005 and June 2008, all patients who were admitted to our centre with periacetabular metastatic disease were considered for this study. We included all patients who were pre-morbidly ambulating before disease progression, able to tolerate the extensive surgery and had a life expectancy of more than 3 month. Twelve patients met the inclusion criteria, six males and six females with a mean age of 47 years (range: 24 to $70 y$ ). Primary malignancies were located in the breast in five cases, and one each in the prostate, thyroid and maxillary sinus. Four acetabular defects were due to multiple myeloma.

Periacetabular defects were categorized based on Harrington's classification: Grade I - periacetabular lesion with intact roof, medial wall and lateral cortices; Grade II deficient medial wall; and, Grade III - deficient roof and lateral cortices ${ }^{3}$. Radiological assessment for periacetabular involvement revealed Harrington grade II in one patient, grade III in ten patients and IV in one patient. All patients had a Technetium 99m MDP bone scan for screening of other distant metastases. Ten patients had multiple osseous metastases and two required simultaneous fixation and total hip replacement of the contralateral femur. Four patients had spine metastases, which were controlled by external beam radiotherapy. One patient with breast metastases who initially underwent dynamic hip screw stabilisation required resection and replacement of the proximal femur with endoprosthesis and acetabular reconstruction due to disease progression. One patient with breast carcinoma had pulmonary metastasis with minimal pleural effusion,

All patients were given a single dose of low molecular weight heparin within six hours surgical intervention followed by a daily dose until patient was able to ambulate. Postoperatively, all patients received external beam radiation therapy delivered by a linear accelerator. A total dose of $30 \mathrm{~Gy}$ were given in ten fractions over two weeks. Radiation treatment was generally started within two to four weeks of surgery. Intravenous pamidronate was administered to all patients with breast, multiple myeloma and prostatic carcinoma.

\section{Surgical technique}

Harrington originally described the use of a postero-lateral approach without femoral trochanteric osteotomy in lateral 
decubitus position ${ }^{3}$, however we used a two-window exposure technique in order to address the extensive acetabular defect. The anterior window approach is a modification of Hardinge, where the posterior gluteus medius is retained. The posterior window is accessed by dividing the piriformis tendon to expose the entire posterosuperior acetabular region and the greater sciatic foramen. This window is an important guide for the direction of Steinmann pin insertion to the sacroiliac joint and proper seating of the anti-protrusio cage.

The use of a Steinmann pin for reconstruction is a modification by Henry Finn where the three columns of acetabulum are bridged to ensure stable reconstruction ${ }^{5}$. The medial column is supported by a cross pin from the posterosuperior acetabulum to the superior pubic rami and the posterior column through pinning from iliac crest to ischium. Two threaded pins fastened to the sacroiliac joint further stabilize the superior acetabulum. We performed cross pin reconstructions for four extensive acetabular defects, and the remaining were reconstructed with augmentation of antiprotrusio cages using multiple long screws directed to the sacroilliac joint. Anti-protrusio cages with polyethylene linear were cemented in place incorporating the threaded pins to achieve stable fixation.

\section{RESULTS}

Functional evaluations using the Musculoskeletal Tumour Society scoring system were good or satisfactory in all patients. Six patients had an excellent score. All patients were able to ambulate postoperatively, although five required the aid of a walking stick or frame after prolonged walking due to muscle weakness. Eleven patients were ambulating pain free with a mean Musculoskeletal Tumour Society (MSTS) functional score of 80 (range, 68 to 86). One patient with pre-operative pulmonary effusion died peri-operatively due to pulmonary embolism.

Palliative analgesic requirements were lower postoperatively compared to preoperative administration. Preoperatively, most patients were bedridden (7 of 12) and required a combination of non-steroidal anti-inflammatory drugs and opiods with visual analogue scale for pain ranging from six to nine. The pain score was reduced after surgery and most of the patient did not require regular analgesics (Table I). All surviving patients started ambulating within two weeks of surgery. At 12 months post-surgery, six patients were still alive. The mean duration of survival was 10 months. There was no post-operative infection, dislocation or failures of reconstruction noted in the current series.

\section{DISCUSSION}

Destruction of the pelvis is a common problem in patients with skeletal metastasis ${ }^{2,3}$, and the pain and disability secondary to pelvic destruction are often very significant. Peri-acetabular destruction is associated with severe hip pain and dysfunction, and may not be relieved by limiting weight bearing and radiation treatment alone. These lesions need stabilization of the joint in addition to pain relief. Metastatic tumour in the peri-acetabular region over time often leads to gradual development of protrusio acetabulum ${ }^{2,3,5}$, due to the disruption of weight transfer from the femoral head to the superior acetabular dome and posterior column, resulting in the protrusion being directed superiorly and medially.

The goals of surgical reconstruction are to relieve pain and improve hip function allowing early mobilization with immediate stability in the postoperative period. In cases where the structural integrity of the acetabulum is still maintained, these goals are best accomplished by reconstruction with cemented arthroplasty. When there is extensive periacetabular destruction, the acetabular component fixation requires a special surgical technique. In these cases, conventional total hip replacement will fail because the structurally deficient bone is not adequate for prevention of acetabular component migration and loosening. There are several other options for reconstructive techniques to manage periacetabular metastases. These include resection followed by saddle prosthesis and McMinn cup with custom periacetabular prosthesis. However these surgeries are complex and the risk of complications are high.

Harrington described an alternative technique to improve fixation and allow weight transfer to the entire pelvis ${ }^{3}$. This reconstruction method includes placement of multiple threaded Steinmann pis between the intact superior acetabulum and the sacroiliac joint. An acetabular antiprotrusio cage is placed in conjunction with the pins, and the entire construct is cemented together with the polyethylene liner. Not only does this provide a means of transferring the weight bearing stresses from the acetabular roof to the spine, it also provides a long lasting stable fixation for the acetabular component. Stable reconstruction was achieved in all patients in the current series and all were able to ambulate pain-free within two weeks of surgery. Further, there were no reconstruction failures despite progression of the metastatic disease (Figure 4). The results of this study are comparable with other series using this technique for acetabular reconstruction ${ }^{6,7,8}$. The early result of functional scoring system with an average of $80 \%$ is considered to be good and we anticipate that the score to became lower as the disease progressed.

The risk of complications with anaesthesia was quite high in this group of patients ${ }^{6,910}$. They commonly present with complications of the carcinomatosis such as metastasis to the lungs, and/or invasion or infiltration to blood vessels and malnutrition. Patients may also have multiple pulmonary metastases complicated with effusions and pneumothorax. All of these factors contribute to prolonged operating time, massive blood loss and postoperative pneumonia. The 


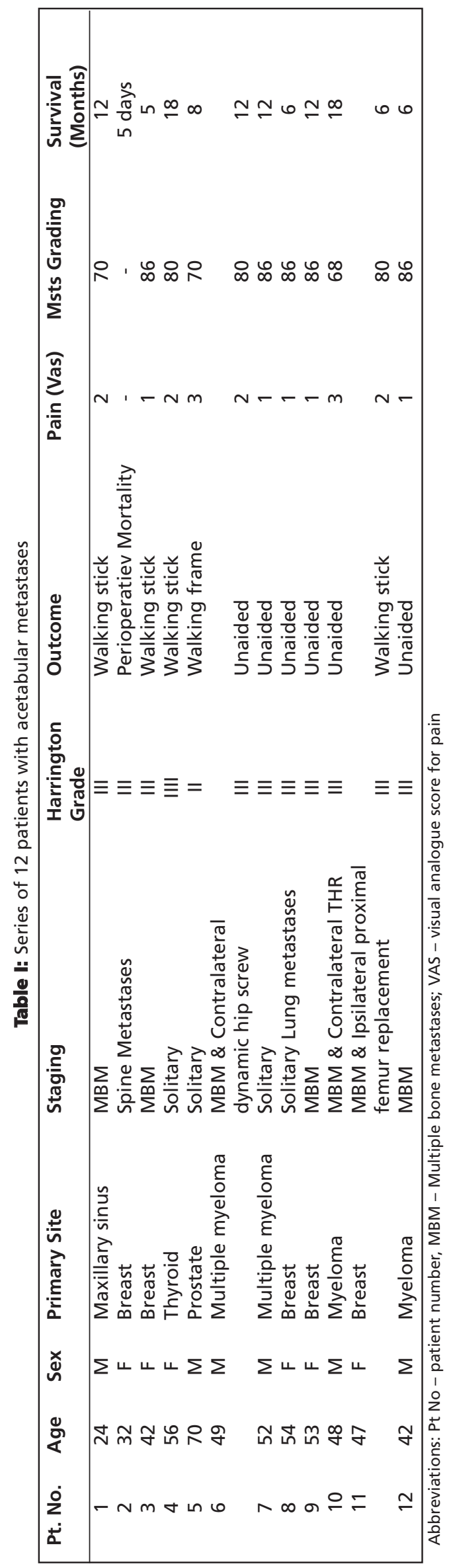




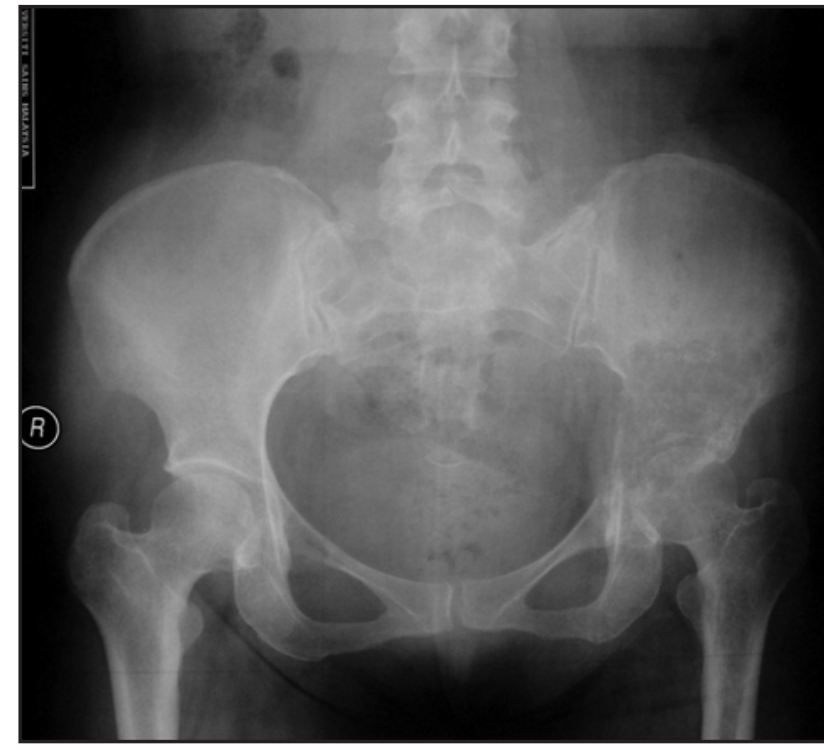

Fig. 1A: Left hip radiograph of a multiple myeloma patient showing class III acetabular destruction.
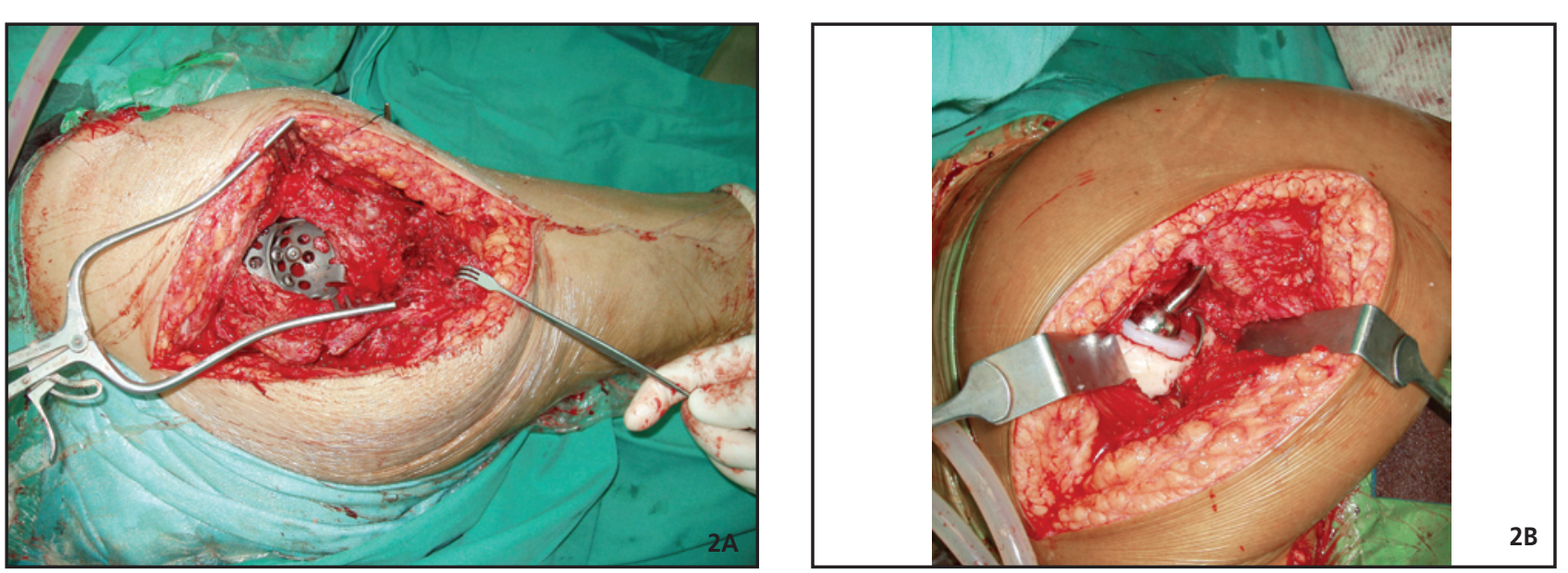

Fig. 2A\&B : Two window approach for metastatic acetabular defect. The acetabular defect was reconstructed with anti-potrusio titanium cage and cemented hip replacement.

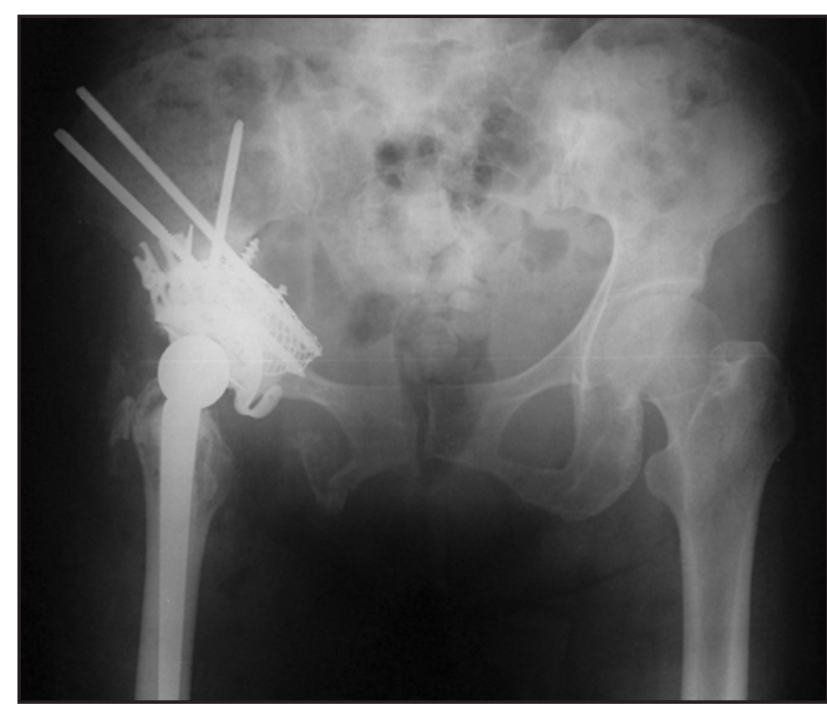

Fig. 3: Radiograph showing reconstruction of metastatic acetabular defect with threaded pins and anti-potrusio cage cemented. Multiple femur metastatic lesions reconstructed with long cemented stem.

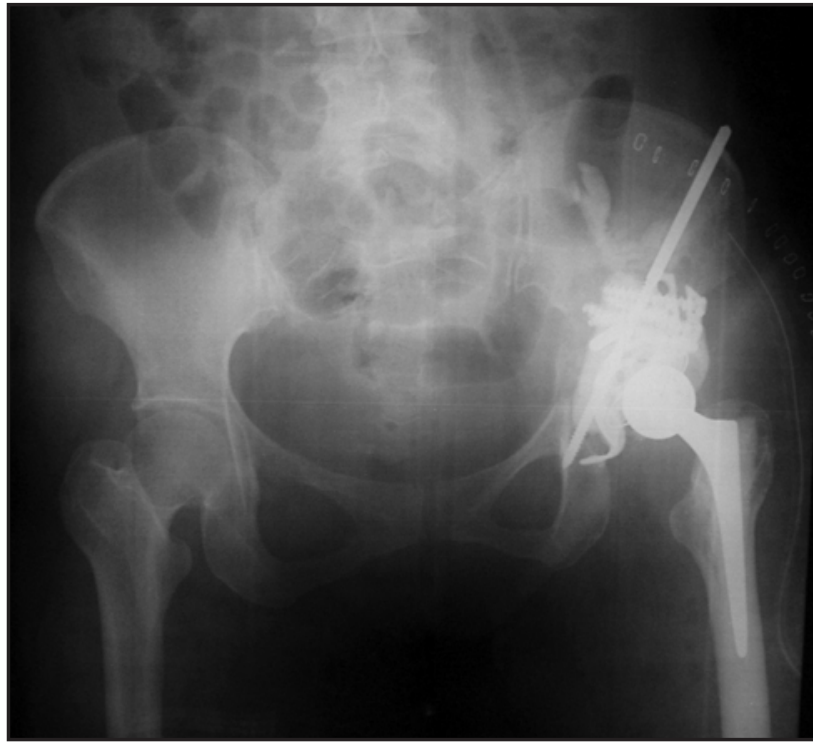

Fig. 1B: Radiograph showing a left hip replacement with multiple threaded pins and long screws with a flanged antipotrusio acetabular cage.

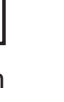


prolonged preoperative immobilization due to fracture entails a high risk of deep vein thrombosis ${ }^{6,9,10}$. This explains the single case of perioperative mortality (Table I, Patient 2) due to pulmonary embolism despite administration of low molecular weight of heparin in all cases .

Postoperative external beam radiation was administered in all surviving patients, a treatment paradigm supported by results of a study Townsend et al where they found that $15 \%$ of patients treated with surgery alone required a second orthopaedic procedure due of increasing pain and radiographic loosening of prosthesis. In contrast, in patients treated with postoperative irradiation, only $3 \%$ require a second operative procedure ${ }^{4}$.

Prophylactic use of biphosphonate has been shown to decrease the actual incidence of bone metastases, minimizing orthopaedic events and oncologic emergency due to malignant hypercalcaemia ${ }^{8}$. Pamidronate has previously been used in patients with established osseous metastases from breast cancer, prostate cancer and multiple myeloma, and has been shown to decrease tumour burden and induce remission or healing in osseous lesions without the need for concurrent chemotherapy or radiotherapy ${ }^{11}$. A combination of the surgical procedure with post-operative external irradiation definitively improved the quality of life by early ambulation and pain relief in the current series.

\section{CONCLUSION}

Hip reconstruction using a modified Harrington procedure achieves good pain control and functional outcome in patients with acetabular insufficiency due to metastatic malignant disease. This is essential to ensure good quality of life. 


\section{REFERENCES}

1. Ward WG, Spang J, Howe D. Metastatic disease of the femur, Surgical management, Orthop Clin North Am 2000; 31: 633-45.

2. Wunder JS, Ferguson PC, Griffin AM. Acetabular metastases planning for reconstruction and review of results. Clin Orthop Relat Res 2003; 415S: 187-97.

3. Harrington KD. The management of acetabular insufficiency secondary to metastatic malignant disease. J Bone Joint Surg 1981; 63-A; 653.

4. Townsend PW, Rosenthal HG, Smalley SR. Impact of postoperative radiation therapy and other perioperative factors on outcome after orthopaedic stabilization of impending pathological fractures due to metastatic disease. J Clin Oncol 1994; 12: 2345.

5. Finn HA. Pelvis and acetabulum. In Surgery for bone and soft tissue tumours. Philadelphia: Lippincott-Raven publishers; 1983. 671-82.

6. Kunisada T, Choong PFM. Major reconstruction for periacetabular metastasis: early complication and outcome following surgical treatment in 40 hips. Acta Orthop Scand 2000: 71(6); 585-90.

7. Tillman RM, Myers GJC, Abudu AT, Carter SR, Grimer RJ. The three pin modified "Harrington" procedure for advanced metastatic destruction of the acetabulum. J Bone Joint Surg 2008: 90-B; 84-7.

8. Wangsaturaka P, Asavamongkolkul A, Waikakul S, Phimolsarnti R. The result of surgical management of bone metastasis involving the periacetabular area: Sriraj experience. J Med Assoc Thai 2007: 90; 1006-13.

9. De Sancho MT, Rand JH. Bleeding and thrombotic complication in critically ill patient of cancer. Crit Care Clin 2001; 17(3): 599-622.

10. Choong PFM. Cardiopulmonary complications of intramedullary fixation of long bone metastases. Clin Orthop Relat Res 2003; 415S: 245-53.

11. Matthew RS. Zolendronic acid to prevent skeletal complications in cancer: Corrobrating the experience. Cancer treat Rev. 2005; 31: 519- 25 . 